\title{
Spatial filter by spike timing dependent plasticity
}

\author{
Kazuhisa Fujita \\ From Nineteenth Annual Computational Neuroscience Meeting: CNS*2010 \\ San Antonio, TX, USA. 24-30 July 2010
}

Spike timing dependent plasticity (STDP) is found in various areas of the brain, visual cortex, hippocampus and hindbrain of electric fish, etc. The synaptic modification by STDP depends on time difference between presynaptic spike arrival and postsynaptic firing. If presynaptic neuron fires earlier than postsynaptic neuron dose, synaptic weight is strengthened. If postsynaptic neuron fires earlier than presynaptic neuron dose, synaptic weight is weakened. This learning rule is one example of various rules (hippocampal type). The learning rule of electric fish type is reversed. Changes of synaptic efficiency precisely depend on timing of preand postsynaptic spikes under STDP. Because of this precise dependence, STDP is thought to play the important role in temporal processing of stimuli. Temporal processing by STDP is well known. However, the role of STDP in spatial processing is not enough understood. To investigate spatial processing by STDP, We studied the role in spatial processing on interconnected network with STDP, using computer simulation. In this study, we found two type spatial filter by STDP on interconnected network. One is low pass filter using the learning rule of electric fish type. Another is edge-enhancing filter using the learning rule of hippocampus type.

In this study, we calculated the response of interconnected neural network with STDP learning. The structure of the network is one-dimensional array. The neuron of the network has connection to neighbour neurons.

In the case of learning rule of electric fish type, the network provided the function of low pass filter. The (a)

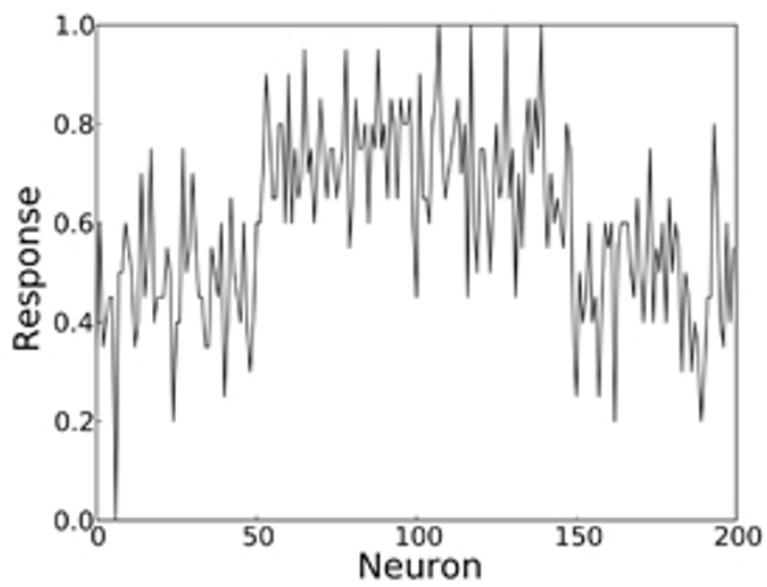

(b)

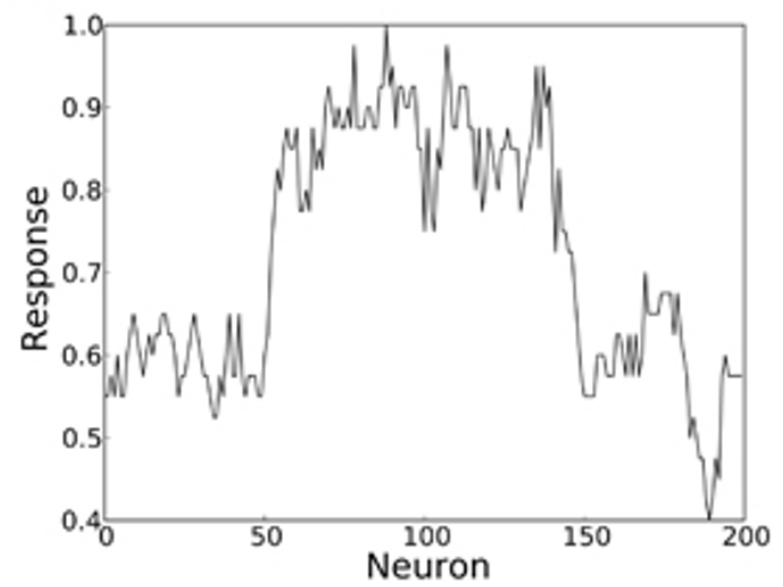

Figure 1 Response of network. Vertical and horizontal line indicates response of the network and neuronal number, respectively. (a) and (b) show the response of the network without and with STDP learning, respectively.

Correspondence: kazu@spikingneuron.net

Department of Computer and Information Engineering, Tsuyama National

Collage of Technology, 654-1 Numa Tsuyama, Okayama Japan 708-8506 
network was stimulated by direct current rectangularly distributed with noise. We examined two cases. In one case, synaptic modification did not occur (Fig 1a). In another case, synaptic modification occurred (Fig 1b). In the first case, Noise presented in response of the network and covered the rectangular form of stimulus. In the second case, noise was eliminated and the rectangular form of stimulus was represented. In the case of learning rule of hippocampal type, the network provided the function of edge-enhancing filter. The network was stimulated by direct current rectangularly distributed. The firing rate of the neuron that represents the edge of stimulus was larger than one of the others. This result shows that the network with hipocampal type STDP plays the role of edge-enhancing filter. These results show that interconnected network with STDP provides the function of spatial filter, and suggest that STDP would plays the important role in spatial processing.
doi:10.1186/1471-2202-11-S1-P103

Cite this article as: Fujita: Spatial filter by spike timing dependent plasticity. BMC Neuroscience 2010 11(Suppl 1):P103.
Submit your next manuscript to BioMed Central and take full advantage of:

- Convenient online submission

- Thorough peer review

- No space constraints or color figure charges

- Immediate publication on acceptance

- Inclusion in PubMed, CAS, Scopus and Google Scholar

- Research which is freely available for redistribution

Submit your manuscript at www.biomedcentral.com/submit 